\title{
The Exponential Pareto Power Series Distribution: Theory and Applications
}

\author{
I. Elbatal \\ Department of Mathematics and Statistics, Al Imam Mohammad Ibn \\ Saud Islamic University (IMSIU), Saudi Arabia \\ iielbatal@imamu.edu.sa \\ Mohamed Zayed \\ Department of Statistics, Mathematics and Insurance \\ Benha University, Egypt \\ mohamedzayed352@yahoo.com \\ Mahdi Rasekhi \\ Department of Statistics, Malayer University \\ Malayer, Iran \\ rasekhimahdi@gmail.com \\ Nadeem Shafique Butt \\ Faculty of Medicine in Rabigh, King Abdulaziz University \\ Jeddah, Saudi Arabia \\ nshafique@kau.edu.sa
}

\begin{abstract}
A new lifetime class of distributions is introduced by compounding the exponential Pareto and power series distributions. The compounding procedure follows the same set-up carried out by Adamidis and Loukas (1998). We obtain several properties of the new class including ordinary and conditional, mean deviations, Bonferroni and Lorenz curves, residual and reversed residual lifes and order statistics. The maximum likelihood estimation procedure is carried out to estimate the model parameters. We present three special models of the proposed class.
\end{abstract}

Keywords: Exponential Pareto Distribution, Power Series Distribution, Maximum Likelihood Estimation, Moments.

\section{Introduction}

Statistical distributions are very useful in describing the real world phenomena. Although a modest number of distributions have been developed, there are always scope for developing distributions, studying their properties which are either more flexible or for fitting real world scenarios. There are always an increased interest among statisticians for developing new and more flexible distributions. As a result, the statistical literature contains many new distributions.

The modeling and analysis of lifetimes is an important aspect of statistical work in a wide variety of technological fields. Several distributions have been proposed in the literature to model lifetime data by compounding some useful lifetime distributions. Adamidis and Loukas (1998) proposed the exponential-geometric $(E G)$ distribution by compounding the exponential distribution with the geometric distribution. Wang (2013) introduced the 
exponentiated Lindley geometric distribution. In a similar manner, the exponential logarithmic $(E L)$ distribution is introduced by Tahmasbi and Rezaei (2008). BarretoSouza et al. (2011) and Lu and Shi (2012) proposed the Weibull-geometric (WG) and Weibull-Poisson (WP) distributions which extend the $E G$ and $E P$ distributions, respectively. Further, Rodrigues et al. (2011) defined the Weibull negative binomial $(W N B)$ distribution which includes the $W G$ and $W P$ distributions as special models.

In the same way, several families of distributions were proposed by compounding some useful lifetime and power series distributions in the last few years. Chahkandi and Ganjali (2009) introduced the exponential power series (EPS) family of distributions. Morais and Barreto-Souza (2011) defined the Weibull power series (WPS) family. Mahmoudi and Jafari (2012) defined the generalized exponential power series (GEPS) distributions. Silva et al. (2013) studied the extended Weibull power series (EWPS) family. Bagheri et al. (2016) proposed the generalized modified Weibull power series distribution.

Al-Kadim and Boshi (2013) introduced a new distribution called the exponential Pareto distribution with cumulative distribution function (CDF) given by

$$
G(x)=1-e^{-\alpha\left(\frac{x}{\mu}\right)^{\lambda}}, x>0 .
$$

The associated probability density function (PDF) is given by

$$
g(x)=\frac{\alpha \lambda}{\mu}\left(\frac{x}{\mu}\right)^{\lambda-1} e^{-\alpha\left(\frac{x}{\mu}\right)^{\lambda}},
$$

where $\alpha>0$ and $\lambda>0$ are shape parameters and $\mu>0$ is a scale parameter.

In this paper, we propose and study a new family of exponential Pareto power series (EPPS) models obtained by compounding the exponential Pareto and power series distributions. The new class contains several lifetime models as special cases such the complementary exponential Pareto geometric $(C E P G)$, exponential Pareto Poisson $(E P P)$, exponential Pareto binomial $(E P B)$ and exponential Pareto logarithmic $(E P L)$ models.

Consider a lifetime of a system with $N$ (discrete random variable) components and the positive continuous random variable, say $\mathrm{X}_{\mathrm{i}}$ (the lifetime of ith component), can be denoted by the non-negative random variable, $X_{(1)}=\min \left(X_{1}, X_{2} \ldots, X_{N}\right)$ and $X_{(n)}=$ $\max \left(X_{1}, X_{2} \ldots, X_{N}\right)$ based on whether the components are series or parallel. By taking a system with parallel components in which the random variable $\mathrm{N}$ has the power series distributions and the random variable $\mathrm{X}_{\mathrm{i}}$ follows the exponential Pareto $(E P)$ distribution.

In addition to the new class of distributions is well-motivated for industrial applications and biological organisms studies. For example, consider the time to relapse of cancer under the first-activation scheme. Suppose that the number, say $N$, of carcinogenic cells for an individual left active after the initial treatment follows a power series distribution and let $X_{i}$ be the time spent for the $\mathrm{i}_{\text {th }}$ carcinogenic cell to produce a detectable cancer mass, for $i \geq 1$. If $\left\{X_{i}\right\}, i \geq 1$ is a sequence of iid $E P$ random variables independent of $N$, then the time to relapse of cancer of a susceptible individual can be modeled by the EPPS class of distributions. Second example suppose that the hazard of a component (item) occurs due to the presence of an unknown number, say $N$, of initial defects of the 
same kind, which can be identifiable only after causing hazard and are repaired perfectly. Define by $X_{i}$ the time to the hazard of the device due to the $i_{t h}$ defect, for $i \geq 1$. If we assume that the $X_{i}{ }^{\prime}$ s are independent and identically distributed (iid) EP random variables independent of $N$, which follows a power series distribution, then the time to the first hazard is appropriately modeled by the EPPS family. Further, the first activation scheme may be questionable for certain diseases. Consider that the number $N$ of latent factors that must all be activated by failure follows a power series distribution and assume that $X_{i}$ represents the time of resistance to a disease manifestation due to the ith latent factor has the EP model. In the last-activation scheme, the failure occurs after all $N$ factors have been activated. So, the new class of distributions is able for modeling the time to the failure under last-activation scheme.

The remainder of our paper is organized as follows: In Section 2, we define the new EPPS class of distributions. Some statistical properties of the EPPS distribution including the ordinary and conditional, mean deviations, residual and reversed residual lifes and order statistics are obtained In Section 3. Maximum likelihood estimates of the unknown parameters are presented in Section 4. In Section 5, we investigate three special cases of the EPPS class of distributions. In Section 6, we demonstrate the flexibility and applicability of one special model using a real data set. We provide some concluding remarks in Section 7.

\section{The new class}

Consider a discrete random variable, $N$, with a power series probability mass function (pmf) defined by

$$
P^{n}=P(N=n)=\frac{a_{n} \theta^{n}}{C(\theta)}, n=1,2, \ldots,
$$

where $a_{n} \geq 0$ depends only on $n, C(\theta)=\sum_{n=1}^{\infty} a_{n} \theta^{n}$ and $\theta>0$ is such that $C(\theta)$ is chosen in a way such that $C(\theta)$ is finite and its first, second and third derivatives with respect to $\theta$ are defined and shown by $C^{\prime}(),. C^{/ /}($.$) and C^{/ / /}($.$) , respectively. Let X_{1}, X_{2}$ , $X_{N}$ be iid random variables having the $E P$ distribution (2).

Let $X_{(1)}=\min \left\{X_{i}\right\}_{i=1}^{N}$, then the conditional CDF of $X_{(1)} \mid N=n$ is given by

$$
G_{X_{(1)} \mid N=n}(x)=1-[1-G(x)]^{n}=1-e^{-n \alpha\left(\frac{x}{\mu}\right)^{\lambda}}
$$

which has a $E P$ distribution with parameters $\lambda, \mu$ and $n \alpha$. Then, we get

$$
P\left(X_{(1)} \leq x, N=n\right)=\frac{a_{n} \theta^{n}}{C(\theta)}\left[1-e^{-n \alpha\left(\frac{x}{\mu}\right)^{\lambda}}\right], x>0, n \geq 1 .
$$

The EPPS family of distributions is defined by the marginal CDF of $X_{(1)}$ (for $x>0$ ) as

$$
F(x)=1-\frac{C\left[\theta e^{-\alpha\left(\frac{x}{\mu}\right) \lambda}\right]}{C(\theta)} \text {. }
$$

The corresponding survival function reduces (for $x>0$ ) to

$$
\bar{F}(x)=\frac{C\left[\theta e^{-\alpha\left(\frac{x}{\mu}\right)^{\lambda}}\right]}{C(\theta)} .
$$


The associated PDF of the EPPS family is given (for $x>0$ ) by

$$
f(x)=\theta g(x) \frac{C^{\prime}[\theta-\theta G(x)]}{C(\theta)}=\frac{\theta \alpha \lambda}{\mu}\left(\frac{x}{\mu}\right)^{\lambda-1} e^{-\alpha\left(\frac{x}{\mu}\right)^{\lambda}} \frac{C^{\prime}\left[\theta \mathrm{e}^{\left.-\alpha\left(\frac{x}{\mu}\right)^{\lambda}\right]}\right.}{C(\theta)} \text {. }
$$

We denote a random variable $X$ with $\operatorname{PDF}(5)$ by $X \sim E P P S(\alpha, \lambda, \mu, \theta)$.

The hazard rate function of $X$ is

$$
h(x)=\frac{\theta \alpha \lambda}{\mu}\left(\frac{x}{\mu}\right)^{\lambda-1} e^{-\alpha\left(\frac{x}{\mu}\right)^{\lambda}} \frac{C^{\prime}\left(\theta e^{-\alpha\left(\frac{x}{\mu}\right)^{\lambda}}\right)}{C\left(\theta e^{\left.-\alpha\left(\frac{x}{\mu}\right)^{\lambda}\right)} .\right.}
$$

This new class of distributions contains several lifetime models distributions which has been introduced and studied in the literature. Table 1 lists some special models that can be derived from the EPPS distribution:

Table 1: Sub-models from the $E P P S$ distribution

\begin{tabular}{|c|c|c|c|c|l|}
\hline Model & $\boldsymbol{C}(\boldsymbol{\theta})$ & $\boldsymbol{\alpha}$ & $\boldsymbol{\mu}$ & $\boldsymbol{\lambda}$ & \multicolumn{1}{|c|}{ Author(s) } \\
\hline $\boldsymbol{W P S}$ & - & - & 1 & - & Morais and Barreto-Souza (2011) \\
\hline $\boldsymbol{E P S}$ & - & - & 1 & 1 & Chahkandi and Ganjali (2009) \\
\hline $\boldsymbol{R P \boldsymbol { S }}$ & - & 1 & - & - & Morais and Barreto-Souza (2011) \\
\hline $\boldsymbol{E P \boldsymbol { G }}$ & $\theta(1-\theta)^{-1}-$ & - & - & - & \\
\hline $\boldsymbol{W G}$ & $\theta(1-\theta)^{-1}-$ & - & 1 & - & Barreto-Souza et al. (2011) \\
\hline $\boldsymbol{E G}$ & $\theta(1-\theta)^{-1}$ & - & 1 & 1 & Adamidis and Loukas (1998) \\
\hline $\boldsymbol{R G}$ & $\theta(1-\theta)^{-1}$ & - & 1 & 2 & \\
\hline $\boldsymbol{E P P}$ & $e^{\theta}-1$ & - & - & - & \\
\hline $\boldsymbol{W P}$ & $e^{\theta}-1$ & - & 1 & - & Lu and Shi (2012) \\
\hline $\boldsymbol{E P}$ & $e^{\theta}-1$ & - & 1 & 1 & \\
\hline $\boldsymbol{R P}$ & $e^{\theta}-1$ & - & 1 & 2 & \\
\hline $\boldsymbol{E P \boldsymbol { B }}$ & $(\theta+1)^{m}-1$ & - & - & - & \\
\hline $\boldsymbol{W B}$ & $(\theta+1)^{m}-1$ & - & 1 & 1 & \\
\hline $\boldsymbol{E B}$ & $(\theta+1)^{m}-1$ & - & 1 & 1 & \\
\hline $\boldsymbol{R B}$ & $(\theta+1)^{m}-1$ & - & 1 & 2 & \\
\hline $\boldsymbol{E P} \boldsymbol{L}$ & $-\log (1-\theta)$ & - & - & - & \\
\hline $\boldsymbol{W} \boldsymbol{L}$ & $-\log (1-\theta)$ & - & 1 & - & \\
\hline $\boldsymbol{E} \boldsymbol{L}$ & $-\log (1-\theta)$ & & 1 & 1 & \\
\hline $\boldsymbol{R} \boldsymbol{L}$ & $-\log (1-\theta)$ & & 1 & 2 & \\
\hline
\end{tabular}

Abbreviations: $R=$ Rayleigh, $E=$ Exponential, $L=$ Logarithmic and $B=$ Binomial.

Remark 1: If $X_{(n)}=\max X_{i=1}^{N}$ then the CDF of $X_{(1 n)}$ is given by

$$
F_{X_{(n)}}(x)=\frac{C(\theta G(x))}{C(\theta)}=\frac{C\left(\theta\left(1-e^{-\alpha\left(\frac{x}{\mu}\right)^{\lambda}}\right)\right)}{C(\theta)}, x>0
$$


and

$$
f(x)=\frac{\theta \alpha \lambda}{\mu}\left(\frac{x}{\mu}\right)^{\lambda-1} e^{-\alpha\left(\frac{x}{\mu}\right)^{\lambda}} \frac{C^{\prime}\left\{\theta\left[1-e^{-\alpha\left(\frac{x}{\mu}\right)^{\lambda}}\right]\right\}}{C\left\{\theta\left[1-e^{-\alpha\left(\frac{x}{\mu}\right)^{\lambda}}\right]\right\}}
$$

Remark 2: The EP distribution with parameters $\alpha, \mu$ and $n \lambda$ is a limiting case of the $E P P S$ class of distributions when $\theta \rightarrow 0^{+}$.

\section{Proof:}

Using $C(\theta)=\sum_{n=1}^{\infty} a_{n} \theta^{n}$ in (4), we have that

$$
\lim _{\theta \rightarrow 0^{+}} F(x)=\lim _{\theta \rightarrow 0^{+}} 1-\lim _{\theta \rightarrow 0^{+}} \frac{\sum_{n=1}^{\infty} a_{n}\left(\theta^{-\alpha\left(\frac{x}{\mu}\right)^{\lambda}}\right)^{n}}{\sum_{n=1}^{\infty} a_{n} \theta^{n}}
$$

Using L'Hôpital's rule, it follows that

$$
\lim _{\theta \rightarrow 0^{+}} F(x)=\lim _{\theta \rightarrow 0^{+}} 1-\lim _{\theta \rightarrow 0^{+}} \frac{a_{1} e^{-\alpha\left(\frac{x}{\mu}\right)^{\lambda}}+\sum_{n=2}^{\infty} n a_{n} \theta^{n-1} e^{-n \alpha\left(\frac{x}{\mu}\right)^{\lambda}}}{a_{1}+\sum_{n=2}^{\infty} n a_{n} \theta^{n-1}}=1-e^{-\alpha\left(\frac{x}{\mu}\right)^{\lambda}} .
$$

Proposition 1: The densities of EPPS class can be expressed as an infinite number of linear combination (mixture) of density of order statistics of $E P$ densities with parameters $\lambda, \mu$ and $n \alpha$.

Proof: Using $C^{\prime}(\theta)=\sum_{n=1}^{\infty} n a_{n} \theta^{n-1}$, we have

$$
\begin{aligned}
& f(x)=\frac{\theta \alpha \lambda}{\mu}\left(\frac{x}{\mu}\right)^{\lambda-1} e^{-\alpha\left(\frac{x}{\mu}\right)^{\lambda} \frac{C^{\prime}\left[\theta e^{-\alpha\left(\frac{x}{\mu}\right)^{\lambda}}\right]}{C(\theta)}} \\
& =\sum_{n=1}^{\infty} \frac{a_{n} \theta^{n}}{C(\theta)} \frac{n \alpha \lambda}{\mu}\left(\frac{x}{\mu}\right)^{\lambda-1} e^{-n \alpha\left(\frac{x}{\mu}\right)^{\lambda}} \\
& =\sum_{n=1}^{\infty} P(N=n) g_{(1)}(x ; n) .
\end{aligned}
$$

where $g_{(1)}(x ; n)$ is the PDF of $X_{(1)}=\min \left(X_{1}, X_{2}, X_{n}\right)$, given by

$$
g_{(1)}(x ; n)=n g(x)[1-G(x)]^{n-1}=\frac{n \alpha \lambda}{\mu}\left(\frac{x}{\mu}\right)^{\lambda-1} e^{-n \alpha\left(\frac{x}{\mu}\right)^{\lambda}}
$$

i.e: $g_{(1)}(x ; n)$ is PDF of $E P$ distribution with parameters $\lambda, \mu$ and $n \alpha$. Thus

$$
f(x)=\sum_{n=1}^{\infty} P(N=n) g_{(1)}(x ; n)=\sum_{n=1}^{\infty} P^{n} g(x ; \lambda, \mu, n \alpha) .
$$

Hence, we can obtain some mathematical properties of the EPPS distribution, from those properties of the exponential Pareto distribution.

\section{Statistical properties}

In this section, we derive some properties of the EPPS distribution including ordinary and conditional moments, moment generating function, mean deviations, Bonferroni and Lorenz curves, residual and reversed residual life functions and order statistics. 


\subsection{Moments}

The $r$ th moment of $X$ follows from (6) as

$$
\begin{aligned}
& \mu_{r}^{\prime}=E\left(X^{r}\right)=\int_{0}^{\infty} x^{r} f(x) d x={ }_{n=1}^{\infty} \sum_{n=1}^{\infty} P^{n} \int_{0}^{\infty} x^{r} g(x ; \lambda, \mu, n \alpha) d x \\
& \sum_{n=1}^{\infty} P^{n} n \alpha \lambda \mu^{r-1} \int_{0}^{\infty}\left(\frac{x}{\mu}\right)^{r+\lambda-1} e^{-n \alpha\left(\frac{x}{\mu}\right)^{\lambda}} d x \\
& =\sum_{n=1}^{\infty} \frac{a_{n} \theta^{n}}{n^{\frac{r}{\lambda}}} \frac{\Gamma\left(\frac{r}{\lambda}+1\right) \mu^{r}}{C(\theta) \alpha^{\frac{r}{\lambda}}} .
\end{aligned}
$$

The conditional moments for EPPS distribution is given by

$$
\begin{aligned}
v_{s} & =E\left(X^{s} \mid X<t\right)=\int_{0}^{t} x^{s} f(x) d x=\sum_{n=1}^{\infty} P^{n} \int_{0}^{t} x^{s} g(x ; \lambda, \mu, n \alpha) d x \\
& =\frac{\gamma\left(\frac{s}{\lambda}+1, n \alpha\left(\frac{t}{\mu}\right)^{\lambda}\right) \mu^{s}}{C(\theta) \alpha^{\frac{s}{\lambda}}} \sum_{n=1}^{\infty} \frac{a_{n} \theta^{n}}{n^{\frac{s}{\lambda}}}
\end{aligned}
$$

where $\gamma(a, t)=\int_{0}^{t} z^{\alpha-1}(1-z)^{b-1} d z$ is the lower incomplete gamma function.

The moment generating function of EPPS distribution is given by

$$
\begin{aligned}
M_{X}(t) & =E\left(e^{t X}\right)=\sum_{r=0}^{\infty} \frac{t^{r^{\infty}}}{r !_{0}} x^{r} f(x)=\sum_{r=0}^{\infty} \frac{t^{r}}{r !} \mu_{r}^{\prime} \\
& =\sum_{r=0}^{\infty} \frac{t^{r}}{r !} \sum_{n=1}^{\infty} \frac{a_{n} \theta^{n}}{n^{\frac{r}{\lambda}}} \frac{\Gamma\left(\frac{r}{\lambda}+1\right) \mu^{r}}{C(\theta) \alpha^{\frac{r}{\lambda}}}
\end{aligned}
$$

\subsection{Mean deviations}

In statistics, mean deviation about the mean and mean deviation about the median measure the amount of scatter in a population. the mean deviation from the mean is a robust statistic, being more resilient to outliers in a data set than standard deviation . For random variable $X$ with PDF $f(x)$, distribution function $F(x)$, mean $\mu=E(X)$ and $M=$ $\operatorname{Median}(\mathrm{X})$, the mean deviation about the mean and mean deviation about the median, are defined by

and

$$
\delta_{1}(x)=\int_{0}^{\infty}|x-\mu| f(x) d x=2 \mu F(\mu)-2 I(\mu) .
$$

$$
\delta_{2}(x)=\int_{0}^{\infty}|x-M| f(x) d x=2 M F(M)-M+\mu-2 I(M) .
$$

respectively, where

$$
I(z)=\int_{0}^{z} x f(x) d x=\sum_{n=1}^{\infty} P^{n} \frac{\gamma\left(\frac{1}{\lambda}+1, n \alpha\left(\frac{z}{\mu}\right)^{\lambda}\right) \mu}{\alpha^{\frac{1}{\lambda}}}
$$


The Bonferroni and Lorenz curves and the Bonferroni and Gini indices have applications in economics, reliability, demography, insurance and medicine. The Bonferroni and Lorenz curves of EPPS distribution are defined, respectively, by

$$
B(p)=\frac{1}{p \mu} \int_{0}^{q} x f(x) d x \text { and } L(p)=\frac{1}{\mu} \int_{0}^{q} x f(x) d x
$$

Using (7), we can write

$$
B(p)=\frac{1}{p \mu}\left[\sum_{n=1}^{\infty} P^{n} \frac{\gamma\left(\frac{1}{\lambda}+1, n \alpha\left(\frac{q}{\mu}\right)^{\lambda}\right) \mu}{\alpha^{\frac{1}{\lambda}}}\right]
$$

and

$$
L(p)=\frac{1}{\mu}\left[\sum_{n=1}^{\infty} P^{n} \frac{\gamma\left(\frac{1}{\lambda}+1, n \alpha\left(\frac{q}{\mu}\right)^{\lambda}\right) \mu}{\alpha^{\frac{1}{\lambda}}}\right]
$$

\subsection{Residual and reversed residual lifes}

Given that a component survives up to time $t \geq 0$, the residual life is the period beyond $t$ until the time of failure and defined by the conditional random variable $X-t \mid X>t$. In reliability, it is well known that the mean residual life function and ratio of two consecutive moments of residual life determine the distribution uniquely. Therefore, the $r$ th order moment of the residual lifetime can be obtained via the general formula

$$
\mu_{r}(t)=E\left((X-t)^{r} \mid X>t\right)=\frac{1}{\bar{F}(t)} \int_{t}^{\infty}(x-t)^{r} f(x) d x, r \geq 1
$$

Applying the binomial series and using (6), we obtain

$$
\begin{aligned}
\mu_{r}(t) & =\frac{1}{\bar{F}(t)} \sum_{n=1}^{\infty} \sum_{d=0}^{r}(-t)^{d}\left(\begin{array}{l}
r \\
d
\end{array}\right) \frac{P^{n} n \alpha \lambda^{\infty}}{\mu^{\lambda}} x^{r+\lambda-d-1} e^{-n \alpha\left(\frac{x}{\mu}\right)^{\lambda}} d x \\
& =\frac{1}{\bar{F}(t)} \sum_{n=1}^{\infty} \sum_{d=0}^{r}(-t)^{d}\left(\begin{array}{l}
r \\
d
\end{array}\right) \frac{n \mu^{\lambda-d}}{(n \alpha)^{\frac{r-d}{\lambda}}} \Gamma\left(\frac{r-d}{\lambda}+1, n \alpha\left(\frac{t}{\mu}\right)^{\lambda}\right),
\end{aligned}
$$

where $\Gamma(a, t)=\int_{t}^{\infty} z^{a-1}(1-z)^{b-1} d z$ is the upper incomplete gamma function.

On the other hand, we analogously discuss the reversed residual life and some of its properties. The reversed residual life can be defined as the conditional random variable $t-X \mid X \leq t$ which denotes the time elapsed from the failure of a component given that its life is less than or equal to $t$.

The $r$ th order moment of the reversed residual life is defined by

$$
m_{r}(t)=E\left((t-X)^{r} \mid X \leq t\right)=\frac{1}{F(t)} \int_{t}^{\infty}(t-x)^{r} f(x) d x, r \geq 1 .
$$

Applying the binomial series and using (6), we can write

$$
\begin{aligned}
m_{r}(t) & =\frac{1}{F(t)} \sum_{n=1}^{\infty} \sum_{d=0}^{r}(-t)^{d}\left(\begin{array}{l}
r \\
d
\end{array}\right) \frac{P^{n} n \alpha \lambda^{t}}{\mu^{\lambda}} x^{r+\lambda-d-1} e^{-n \alpha\left(\frac{x}{\mu}\right)^{\lambda}} d x \\
& =\frac{1}{F(t)} \sum_{n=1}^{\infty} \sum_{d=0}^{r}(-t)^{d}\left(\begin{array}{l}
r \\
d
\end{array}\right) \frac{n \mu^{\lambda-d}}{(n \alpha)^{\frac{r-d}{\lambda}} \Gamma\left(\frac{r-d}{\lambda}+1, n \alpha\left(\frac{t}{\mu}\right)^{\lambda}\right) .}
\end{aligned}
$$




\subsection{Order statistics}

Let $X_{1: n} \leq X_{2: n} \leq \ldots \leq X_{n: n}$ be a random sample of size $n$ from EPPS distribution. The PDF of the $i$ th order statistic $X_{i: n}$, say $f_{i: n}(x), i=1,2, \ldots, n$, is given by

$$
\begin{aligned}
f_{i: n}(x) & =\frac{f(x)}{\beta(i, n-i+1)}[F(x)]^{i-1}[1-F(x)]^{n-i} f(x) \\
& =\frac{f(x)}{\beta(i, n-i+1)}\left[1-\frac{C\left\{\theta e^{-\alpha\left(\frac{x}{\mu}\right)^{\lambda}}\right\}}{C(\theta)}\right]^{i-1}\left\{\frac{C\left[\theta e^{-\alpha\left(\frac{x}{\mu}\right)^{\lambda}}\right]}{C(\theta)}\right\} .
\end{aligned}
$$

where $f(x)$ is the PDF given by (6).

An alternative form for the PDF of the $i$ th order statistic is

$$
f_{i: n}(x)=\frac{1}{\beta(i, n-i+1)} \sum_{n=1}^{\infty} \sum_{j=0}^{n-1}(-1)^{j}\left(\begin{array}{c}
n-i \\
j
\end{array}\right)[F(x)]^{i+j-1} P^{n} g(x, \alpha, \mu, n \lambda) \text {. }
$$

\section{Estimation}

In this section, we determine the maximum likelihood estimates (MLEs) of the parameters of the EPPS distribution from complete samples only. Let $x_{1}, \ldots, x_{n}$ be a random sample of size $n$ from the EPPS distribution given by (6). Let $\phi=(\alpha, \mu, \lambda, \theta)^{T}$ be $p \times 1$ vector of parameters. The total $\log$-likelihood function for $\phi$ is given by

$$
\begin{aligned}
& L=n \log \alpha+n \log \lambda+n \log \theta-n \log \mu-n(\lambda-1) \log \mu+(\lambda-1) \sum_{i=1}^{n} \log x_{i} \\
& -\alpha \sum_{i=1}^{n}\left(\frac{x_{i}}{\mu}\right)^{\lambda}-n \log C(\theta)+\sum_{i=1}^{n} \log C^{\prime}\left[\theta e^{-\alpha\left(\frac{x_{i}}{\mu}\right)^{\lambda}}\right] .
\end{aligned}
$$

The corresponding score function is given by $U_{n}(\phi)=\left(\frac{\partial L}{\partial \alpha}, \frac{\partial L}{\partial \mu}, \frac{\partial L}{\partial \lambda}, \frac{\partial L}{\partial \theta}\right)^{T}$, where

$$
\begin{aligned}
& \frac{\partial L}{\partial \alpha}=\frac{n}{\alpha}-\sum_{i=1}^{n}\left(\frac{x_{i}}{\mu}\right)^{\lambda}-\theta \sum_{i=1}^{n}\left(\frac{x_{i}}{\mu}\right)^{\lambda} e^{-\alpha\left(\frac{x_{i}}{\mu}\right)^{\lambda}} \frac{C^{/ /}\left[\theta e^{-\alpha\left(\frac{x_{i}}{\mu}\right)^{\lambda}}\right]}{C^{\prime}\left[\theta e^{-\alpha\left(\frac{x_{i}}{\mu}\right)^{\lambda}}\right]}, \\
& \frac{\partial L}{\partial \mu}=\frac{-n}{\mu}-\frac{n(\lambda-1)}{\mu}-\frac{\alpha \lambda}{\mu^{2}} \sum_{i=1}^{n}\left(\frac{x_{i}}{\mu}\right)^{\lambda-1}-\frac{\theta \alpha \lambda}{\mu^{2}} \sum_{i=1}^{n}\left(\frac{x_{i}}{\mu}\right)^{\lambda-1} \frac{C^{/ /}\left[\theta e^{-\alpha\left(\frac{x_{i}}{\mu}\right)^{\lambda}}\right]}{C^{\prime}\left[\theta e^{-\alpha\left(\frac{x_{i}}{\mu}\right)^{\lambda}}\right]} \\
& \frac{\partial L}{\partial \lambda}=\frac{n}{\lambda}-n \log \mu+\sum_{i=1}^{n} \log x_{i}-\alpha \sum_{i=1}^{n}\left(\frac{x_{i}}{\mu}\right)^{\lambda} \log \left(\frac{x_{i}}{\mu}\right) \\
& -\theta \alpha \sum_{i=1}^{n}\left(\frac{x_{i}}{\mu}\right)^{\lambda} e^{-\alpha\left(\frac{x_{i}}{\mu}\right)^{\lambda}} \frac{C^{/ /}\left[\theta e^{-\alpha\left(\frac{x_{i}}{\mu}\right)^{\lambda}}\right]}{C^{/}\left[\theta e^{-\alpha\left(\frac{x_{i}}{\mu}\right)^{\lambda}}\right]} \log \left(\frac{x_{i}}{\mu}\right)
\end{aligned}
$$

and

$$
\frac{\partial L}{\partial \theta}=\frac{n}{\theta}-n \frac{C^{\prime}(\theta)}{C(\theta)}+\sum_{i=1}^{n} e^{-\alpha\left(\frac{x_{i}}{\mu}\right)^{\lambda}} \frac{C^{/ /}\left[\theta e^{-\alpha\left(\frac{x_{i}}{\mu}\right)^{\lambda}}\right]}{C^{\prime}\left[\theta e^{-\alpha\left(\frac{x_{i}}{\mu}\right)^{\lambda}}\right]}
$$


The maximum likelihood estimator of $\phi$, say $\hat{\phi}$, is obtained by solving the nonlinear system $U_{n}(\phi)=0$. The solution of this nonlinear system of equation has not a closed form. For interval estimation and hypothesis tests on the model parameters, we require the information matrix.

\section{Special models}

In this section, we provide three special cases of the EPPS class of distributions.

\subsection{Exponential Pareto geometric (EPG) distribution}

The $E P G$ distribution arises by taking $a_{n}=1$ and $C(\theta)=\theta(1-\theta)^{-1},(0<\theta<1)$. The $\mathrm{CDF}$ and PDF of the $E P G$ distribution are given by

and

$$
F(x)=\frac{(1-\theta) e^{-\alpha\left(\frac{x}{\mu}\right)^{\lambda}}}{1-\theta e^{-\alpha\left(\frac{x}{\mu} \lambda^{\lambda}\right.}}
$$

$$
f(x)=\frac{(1-\theta) \frac{\alpha \lambda}{\mu}\left(\frac{x}{\mu}\right)^{\lambda-1} e^{-\alpha\left(\frac{x}{\mu}\right)^{\lambda}}}{\left[1-\theta e^{-\alpha\left(\frac{x}{\mu}\right)^{\lambda}}\right]^{2}},
$$

respectively. The PDF plots of the EPG model are displayed in Figure 1.
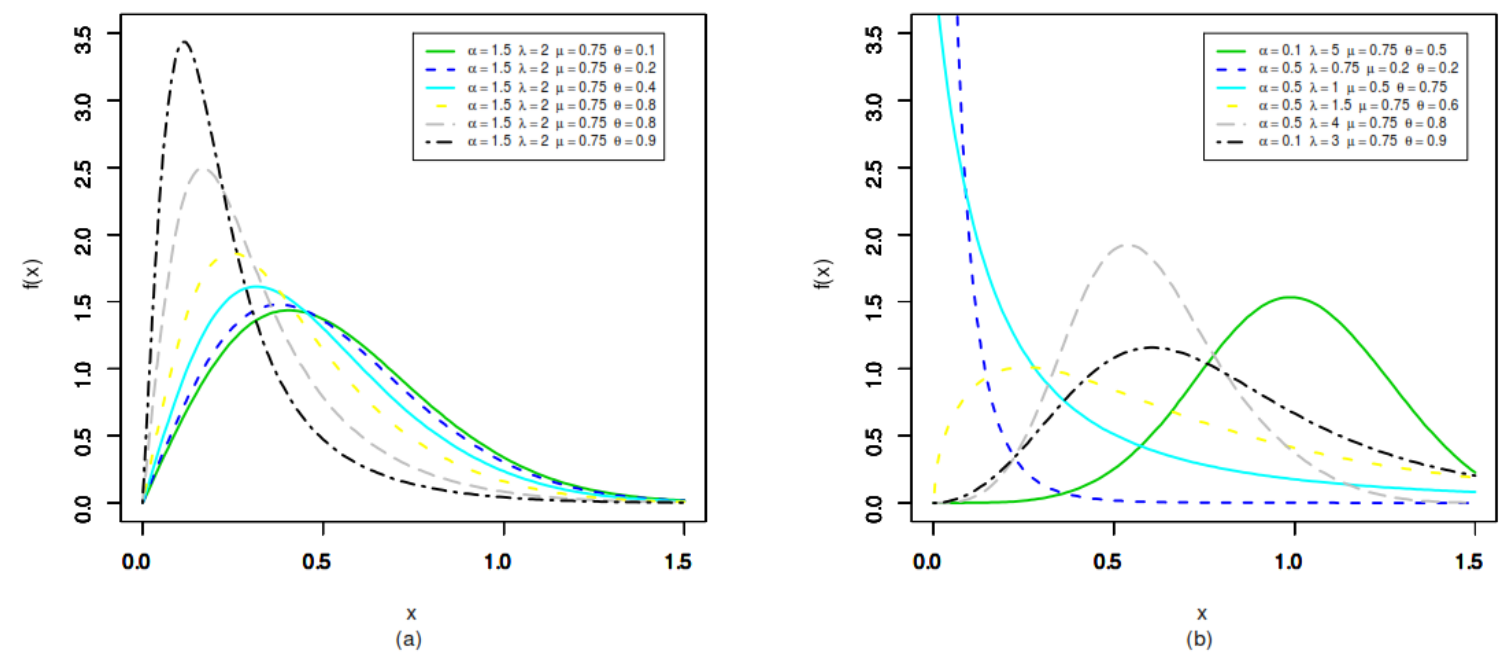

Figure 1: Some possible shapes for the EPG PDF

\subsection{Exponential Pareto Poisson (EPP) distribution}

The EPP distribution arises by taking $a_{n}=n !^{-1}$ and $C(\theta)=e^{\theta}-1, \theta>0$. The CDF and PDF of EPP distribution are, respectively, given by

$$
F(x)=1-\frac{e^{\theta e^{-\alpha\left(\frac{x}{\mu}\right)^{\lambda}}-1}}{e^{\theta}-1}
$$


and

$$
f(x)=\frac{\theta \frac{\alpha \lambda}{\mu}\left(\frac{x}{\mu}\right)^{\lambda-1} e^{-\alpha\left(\frac{x}{\mu}\right)^{\lambda}} e^{\theta e^{-\alpha\left(\frac{x}{\mu}\right)^{\lambda}}}}{e^{\theta}-1} .
$$

Figure 2 shows some plots for the EPP PDF.
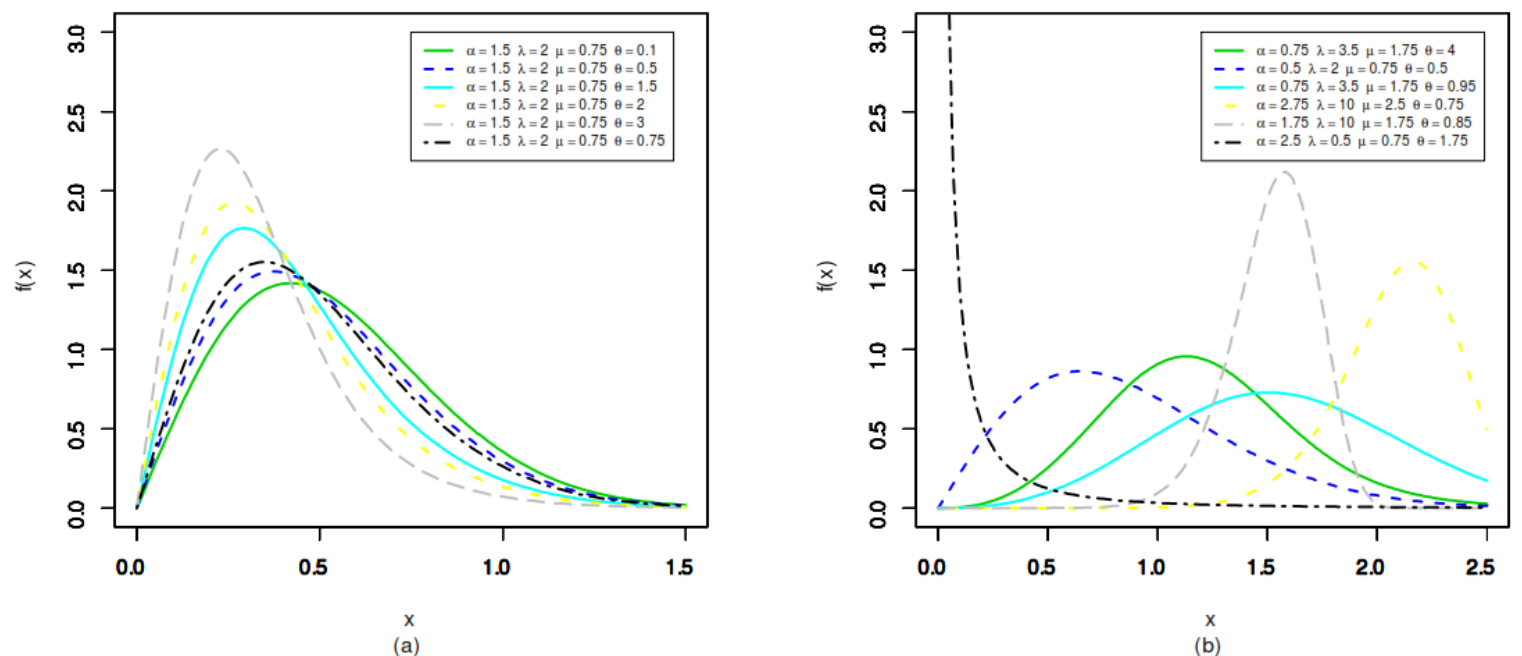

Figure 2: Some possible shapes for the EPP PDF

\subsection{Exponential Pareto logarithmic $(E P L)$ distribution}

The EPL distribution arises by taking $a_{n}=n^{-1}$ and $C(\theta)=-\log (1-\theta), 0<\theta<1$. The CDF and PDF of EPL distribution are given by

$$
\begin{aligned}
& F(x)=1-\log \left[1-\theta e^{-\alpha\left(\frac{x}{\mu}\right)^{\lambda}}\right] / \log (1-\theta) \\
& f(x)=\frac{\alpha \lambda}{-\mu \log (1-\theta)}\left(\frac{x}{\mu}\right)^{\lambda-1} e^{-\alpha\left(\frac{x}{\mu}\right)^{\lambda}}\left[1-\theta e^{-\alpha\left(\frac{x}{\mu}\right)^{\lambda}}\right]^{-1},
\end{aligned}
$$

respectively. The PDF plots of the EPL distribution are given in Figure 3.
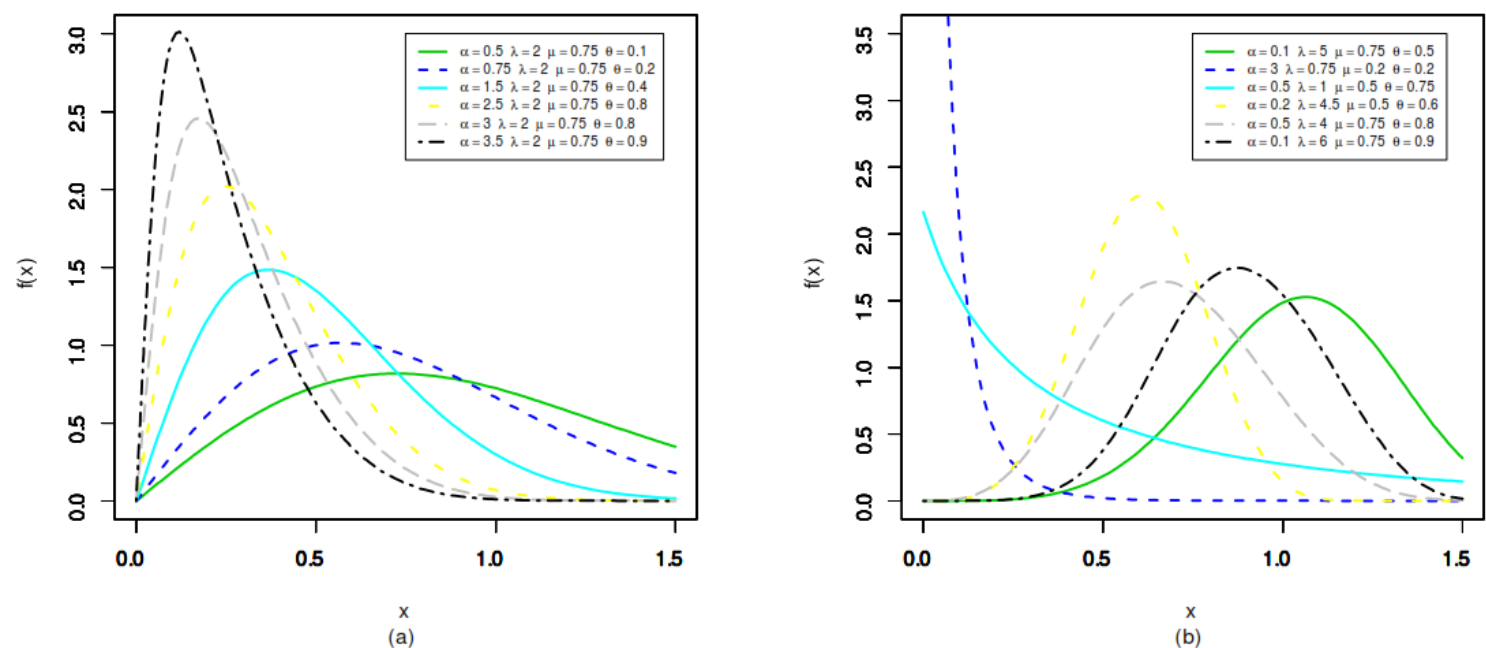

Figure 3: Some possible shapes for the EPL PDF 


\section{Application}

In this section, a real data set is used to demonstrate the flexibility and applicability of the exponential pareto geometric (EPG) distribution over some of generalized pareto (Burr) distributions. In order to compare the models, we used following two criteria:

(a) Cramer Von mises test statistic $\left(W^{*}\right)$.

(b) Anderson and Darling test statistic $\left(A^{*}\right)$.

The EPG distribution is compared to some of Burr generalization with names beta Burr XII distribution, BBXII, (Parnaiba et al., 2011), Kumaraswamy Burr XII, KwBXII, (Parnaiba et al., 2012), beta Burr III, BBIII, (Gomes et al., 2013) and KumaraswamyBurr Type III, KwBIII, (Behairy et al., 2016) distributions. Each distribution was fitted to the data set using the optim() function in R program.

The data set is failure and service times for a particular model windshield given in Table 16.11 of Murthy et al. (2004). The data are as follows: 0.040, 1.866, 2.385, 3.443, 0.301, $1.876,2.481,3.467,0.309,1.899,2.610,3.478,0.557,1.911,2.625,3.578,0.943,1.912$, $2.632,3.595,1.070,1.914,2.646,3.699,1.124,1.981,2.661,3.779,1.248,2.010,2.688$, $3.924,1.281,2.038,2.82,3,4.035,1.281,2.085,2.890,4.121,1.303,2.089,2.902,4.167$, $1.432,2.097,2.934,4.240,1.480,2.135,2.962,4.255,1.505,2.154,2.964,4.278,1.506$, 2.190, 3.000, 4.305, 1.568, 2.194, 3.103, 4.376, 1.615, 2.223, 3.114, 4.449, 1.619, 2.224, $3.117,4.485,1.652,2.229,3.166,4.570,1.652,2.300,3.344,4.602,1.757,2.324,3.376$ and 4.663. The TTT plot (Aarset, 1987) of this sets of data in Figure 4 displays increasing hazards rate function that indicates the appropriateness of the EPG distribution to fit the data sets.

Tables 2 present the maximum likelihood estimate of parameters and mentioned criteria. Figure 5 shows fitted PDFs on histogram of data set. We see that EPG distribution fitted on data better than other rivals.

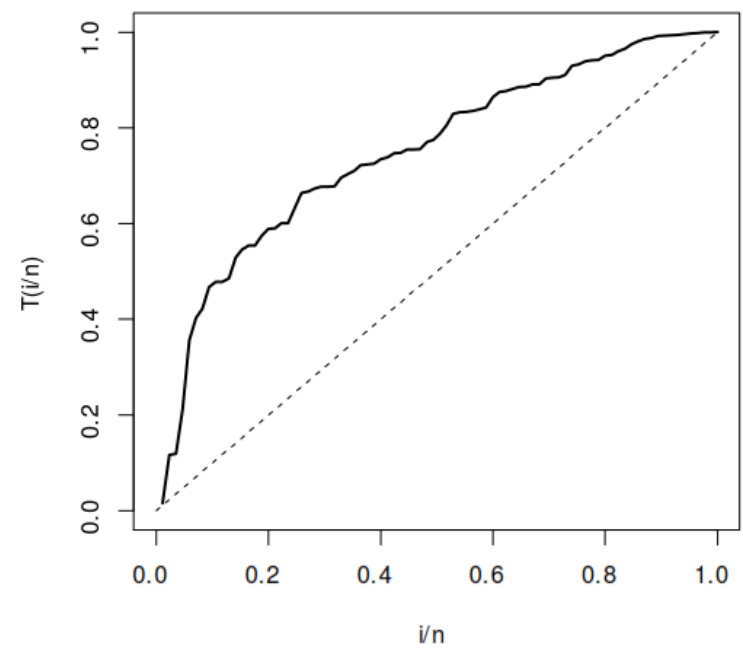

Figure 4: TTT plot of data set 
Table 2: Parameters estimates and goodness-of-fit statistics $\boldsymbol{W}^{*}$ and $\boldsymbol{A}^{*}$

\begin{tabular}{|l|c|c|c|}
\hline Model & Estimates (Standard Error) & $W^{*}$ & $A^{*}$ \\
\hline $\begin{array}{l}E P G \\
(\alpha, \mu, \lambda, \theta)\end{array}$ & $1.550,3.444,2.393,0.001$ & 0.048 & 0.540 \\
\hline $\begin{array}{l}\text { BBXII } \\
(\alpha, \gamma, c, k, a)\end{array}$ & $\begin{array}{c}0.264,1.777,6.252,5.815,5.860 \\
(0.031),(0.358),(0.026),(0.098),(0.027)\end{array}$ & 0.112 & 0.763 \\
\hline $\begin{array}{l}\text { KwBXII } \\
(\alpha, \gamma, c, k, a)\end{array}$ & $\begin{array}{c}0.266,1.881,7.110,5.566,6.007 \\
(0.056),(1.474),(0.002),(7.964),(0.002)\end{array}$ & 0.085 & 0.637 \\
\hline $\begin{array}{l}\text { BBIII } \\
(a, b, \alpha, \beta, s)\end{array}$ & $0.037,2.743,6.090,7.706,3.845$ \\
\hline $\begin{array}{l}\text { KwBIII } \\
(a, b, \alpha, \beta, s)\end{array}$ & $\begin{array}{c}0.140,1.89),(1.778),(1.979),(7.280),(0.419) \\
(0.118),(1.070),(2.154),(1.919),(0.913)\end{array}$ & 0.076 & 0.597 \\
\hline
\end{tabular}

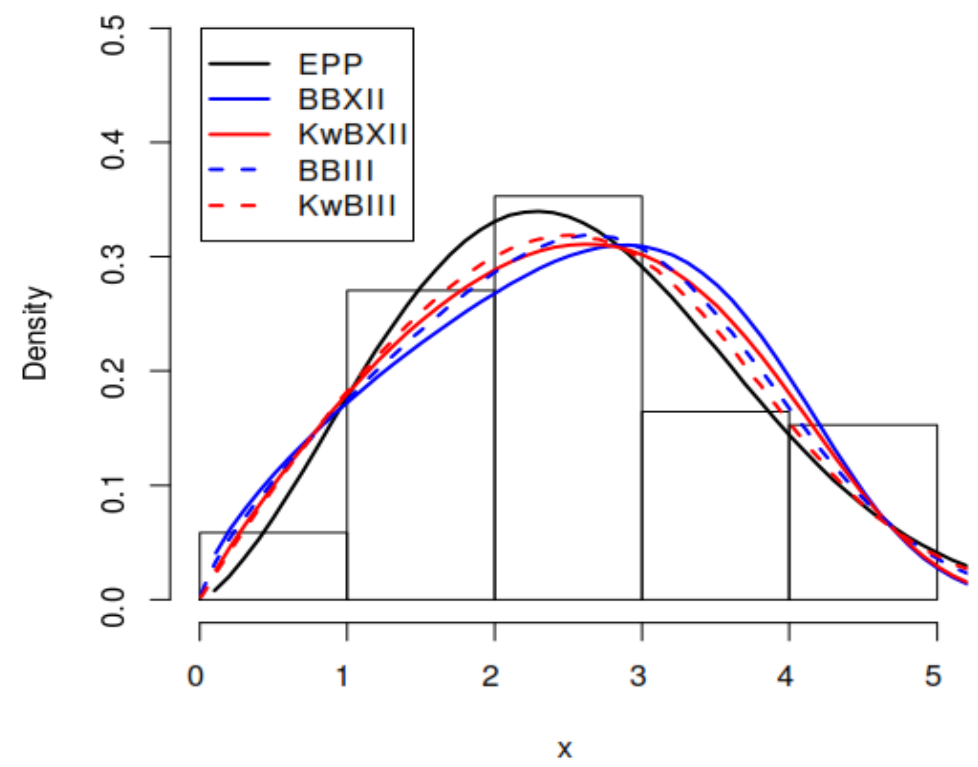

Figure 5: Fitted PDFs on histogram

\section{Conclusion}

We defined a new class called the exponential Pareto power series (EPPS) distributions. Three special models of this family are discussed. We studied its general mathematical properties such as ordinary and conditional, mean deviations, Bonferroni and Lorenz curves, residual and reversed residual lifes and order statistics. The model parameters are estimated using the maximum likelihood method. Its usefulness is illustrated using a real data set.

\section{References}

1. Aarset, A. S. (1987). How to identify a bathtub hazard rate . IEEE Transactions on Reliability, 36, 106-108.

2. Adamidis K. and Loukas, S. (1998). A lifetime distribution with decreasing failure rate. Statistics and Probability Letters, 39, 35- 42. 
3. Al-Kadim, K. A. and Boshi, M. A. (2013). Exponential Pareto distribution. Mathematical Theory and Modeling, 3, 135-146.

4. Bagheri, S. F., Samani, E. B. and Ganjali, M. (2016). The generalized modified Weibull power series distribution: theory and applications. Computational Statistics and Data Analysis, 94, 136-160.

5. Barreto-Souza, W., Morais, A. L. and Cordeiro, G. M. (2011). The Weibullgeometric distribution. Journal of Statistical Computation and Simulation, 81, 645- 657.

6. Behairy, S. M., AL-Dayian, G. R. and EL-Helbawy, A. A. (2016). The Kumaraswamy-Burr type III distribution: properties and estimation. British Journal of Mathematics, 14, 1-21.

7. Chahkandi, M. and Ganjali, M. (2009). On some lifetime distributions with decreasing failure rate. Computational Statistics and Data Analysis, 53, 44334440 .

8. Gomes, A. E., da Silva, C. Q., Cordeiro, G. M. and Ortega, E. M. M. (2013). The beta Burr III model for lifetime data. Brazilian Journal of Probability and Statistics, 27,502-543.

9. Lu, W. and Shi, D. (2012). A new compounding life distribution: theWeibullPoisson distribution. Journal of Applied Statistics, 39, 21-38.

10. Mahmoudi, E. and Jafari, A. A. (2012). Generalized exponential power series distributions. Computational Statistics and Data Analysis, 56, 4047- 4066.

11. Morais, A. L. and Barreto-Souza, W. (2011). A compound family of Weibull and power series distributions. Computational Statistics and Data Analysis, 55, 14101425.

12. Murthy, C. S. R. and Manoj, B. S. (2004). Adhoc wireless networks: architectures and protocols, portable documents. Pearson education.

13. Parnaiba, P. F. P., Ortega, E. M. M., Cordeiro, G. M. and Pescim, R. R. (2011). The beta Burr XII distribution with application to lifetime data. Computational Statistics and Data Analysis, 55, 1118-1136.

14. Parnaiba, P. F. P., Ortega, E. M. M., Cordeiro, G. M. and Pascoa, M. A. R. (2012). The Kumaraswamy Burr XII distribution: theory and practice. Journal of Statistical Computation and Simulation, 82, 1-27.

15. Rodrigues, C. R., Cordeiro, G. M., Demétrio, C. G. and Ortega, E. M. (2011). The Weibull negative binomial distribution. Advances and Applications in Statistics, $22,25-55$.

16. Silva, R. B., Bourguignon, M., Dias, C. R. B. and Cordeiro, G. M. (2013). The compound family of extended Weibull power series distributions. Computational Statistics and Data Analysis, 58, 352- 367.

17. Tahmasbi, R. and Rezaei, S. (2008). A two-parameter lifetime distribution with decreasing failure rate. Computational Statistics and Data Analysis, 52, 38893901.

18. Wang, M. (2013). A new three-parameter lifetime distribution and associated inference. arXiv:1308.4128v1 [stat.ME]. 